Electronic Supplementary information (S) for

\title{
Changes in Particulate and Mineral Associated Organic Carbon with Land Use in Contrasting Soils
}

\author{
Sabina Yeasmin ${ }^{1 \uparrow^{*}}$, Balwant Singh $^{1}$, Cliff T Johnston ${ }^{2}$, Donald L Sparks ${ }^{3}$ and Quan Hua ${ }^{4}$ \\ ${ }^{1}$ Sydney Institute of Agriculture, School of Life and Environmental Sciences, The University of Sydney, \\ Sydney, NSW 2006, Australia, ${ }^{2}$ Crop, Soil and Environmental Sciences, Purdue University, West Lafayette, \\ IN 47907, USA, ${ }^{3}$ Department of Plant and Soil Sciences, University of Delaware, Newark, DE 19716, USA, \\ ${ }^{4}$ Australian Nuclear Science and Technology Organisation, Locked Bag 2001, Kirrawee DC, NSW 2232, \\ Australia \\ ${ }^{\dagger}$ Current address: Department of Agronomy, Bangladesh Agricultural University, Mymensingh-2202, \\ Bangladesh. \\ *Corresponding author: sabinayeasmin@bau.edu.bd
}

\section{Contents of this file}

Table: S1 - S2, and 3-5

Figure: Fig. S1 - Fig. S5

This supporting information provides some additional tables and figures for the manuscript, as well as all of the raw data (Table 3-5) presented in the manuscript. 
1 Table S1 Geological and climatic data for the four sites from where soils samples were collected for the study

\begin{tabular}{|c|c|c|c|c|c|c|c|c|}
\hline \multirow{2}{*}{$\begin{array}{l}\text { WRB soil } \\
\text { order }\end{array}$} & \multirow{2}{*}{$\begin{array}{c}\text { ASC soil } \\
\text { order }\end{array}$} & \multirow{2}{*}{ Latitude } & \multirow{2}{*}{ Longitude } & \multirow{2}{*}{$\begin{array}{c}\text { Average } \\
\text { annual } \\
\text { precipitation } \\
\text { (mm) }\end{array}$} & \multirow{2}{*}{$\begin{array}{c}\text { Average } \\
\text { annual } \\
\text { temperature } \\
\left({ }^{\circ} \mathrm{C}\right)\end{array}$} & \multirow{2}{*}{$\begin{array}{c}\text { Elevation } \\
\text { (m) }\end{array}$} & \multicolumn{2}{|c|}{ Land use } \\
\hline & & & & & & & Native & Cropped \\
\hline Ferralsol & Ferrolsol & $-28^{\circ} 48^{\prime} 55.8^{\prime \prime}$ & $153^{\circ} 24^{\prime} 0.7^{\prime \prime}$ & 1810 & 19.4 & 166 & \multirow{4}{*}{ Open woodland } & \multirow{3}{*}{ Cereal } \\
\hline Luvisol & Chromosol & $-31^{\circ} 5^{\prime} 48.4^{\prime \prime}$ & $150^{\circ} 43^{\prime} 50.7^{\prime \prime}$ & 632 & 17.3 & 404 & & \\
\hline Vertisol & Vertosol & $-31^{\circ} 43^{\prime} 13.1^{\prime \prime}$ & $150^{\circ} 40^{\prime} 54.6^{\prime \prime}$ & 681 & 16.8 & 390 & & \\
\hline Solonetz & Sodosol & $-31^{\circ} 21^{\prime} 11.0^{\prime \prime}$ & $150^{\circ} 4^{\prime} 46.1^{\prime \prime}$ & 638 & 18.4 & 285 & & Cereal + forage crops \\
\hline
\end{tabular}

2 WRB $=$ World Reference Base, ASC = Australian Soil Classification. Climate data source: New South Wales Department of Primary Industries (2014); Australian Bureau of

3 Meteorology, Bureau of Meteorology (2014)

4 
8 Table S2 Mean \pm S.E. $(\mathrm{n}=3)$ values of iron $(\mathrm{Fe})$ and aluminium (Al) extracted using different extractants from bulk soils

\begin{tabular}{|c|c|c|c|c|c|c|c|c|c|}
\hline \multirow{2}{*}{$\begin{array}{c}\text { WRB soil } \\
\text { order }\end{array}$} & \multirow{2}{*}{ Land use } & \multirow{2}{*}{$\begin{array}{l}\text { Depth } \\
(\mathrm{cm})\end{array}$} & \multicolumn{4}{|c|}{ Extractable $\mathrm{Fe}\left(\mathrm{g} \mathrm{kg}^{-1}\right)$} & \multicolumn{3}{|c|}{ Extractable $\mathrm{Al}\left(\mathrm{g} \mathrm{kg}^{-1}\right)$} \\
\hline & & & $\mathrm{Fe}_{\mathrm{Ox}}$ & $\mathrm{Fe}_{\mathrm{DCB}}$ & $\mathrm{Fe}_{\mathrm{Na}-\mathrm{py}}$ & $\mathrm{Fe}_{\mathrm{Ox}}: \mathrm{Fe}_{\mathrm{DCB}}$ & $\mathrm{Al}_{\mathrm{Ox}}$ & $\mathrm{Al}_{\mathrm{DCB}}$ & $\mathrm{Al}_{\text {Na-py }}$ \\
\hline \multirow[t]{3}{*}{ Ferralsol } & Native & $0-10$ & $6.0 \pm 0.04$ & $150 \pm 2.13$ & $9.0 \pm 0.31$ & $0.04 \pm 0.00$ & $3.2 \pm 0.04$ & $12.7 \pm 0.15$ & $3.7 \pm 0.10$ \\
\hline & & $60-70$ & $5.9 \pm 0.54$ & $180 \pm 2.59$ & $3.9 \pm 0.13$ & $0.03 \pm 0.00$ & $3.5 \pm 0.17$ & $15.1 \pm 0.31$ & $1.6 \pm 0.04$ \\
\hline & & $60-70$ & $5.5 \pm 0.38$ & $73 \pm 0.27$ & $1.1 \pm 0.02$ & $0.07 \pm 0.01$ & $3.6 \pm 0.12$ & $8.2 \pm 0.05$ & $0.74 \pm 0.02$ \\
\hline \multirow{2}{*}{ Luvisol } & Cropped & $0-10$ & $2.9 \pm 0.08$ & $15 \pm 1.08$ & $0.54 \pm 0.25$ & $0.19 \pm 0.02$ & $1.1 \pm 0.03$ & $1.4 \pm 0.10$ & $1.0 \pm 0.47$ \\
\hline & & $60-70$ & $2.0 \pm 0.03$ & $15 \pm 2.27$ & $0.34 \pm 0.00$ & $0.14 \pm 0.02$ & $1.5 \pm 0.02$ & $1.6 \pm 0.26$ & $0.32 \pm 0.02$ \\
\hline \multirow[t]{3}{*}{ Vertisol } & Native & $0-10$ & $7.9 \pm 0.21$ & $15 \pm 0.30$ & $0.38 \pm 0.01$ & $0.53 \pm 0.01$ & $2.0 \pm 0.03$ & $1.3 \pm 0.03$ & $0.15 \pm 0.00$ \\
\hline & & $60-70$ & $5.8 \pm 0.30$ & $17 \pm 0.14$ & $0.08 \pm 0.00$ & $0.35 \pm 0.02$ & $1.8 \pm 0.08$ & $1.3 \pm 0.01$ & $0.04 \pm 0.00$ \\
\hline & Cropped & $0-10$ & $4.5 \pm 0.13$ & $14 \pm 0.11$ & $0.23 \pm 0.01$ & $0.32 \pm 0.01$ & $1.9 \pm 0.03$ & $1.4 \pm 0.02$ & $0.14 \pm 0.02$ \\
\hline \multirow{3}{*}{ Solonetz } & & $60-70$ & $1.2 \pm 0.05$ & $22 \pm 0.57$ & $0.12 \pm 0.00$ & $0.05 \pm 0.00$ & $0.71 \pm 0.04$ & $2.4 \pm 0.06$ & $0.13 \pm 0.00$ \\
\hline & Cropped & $0-10$ & $0.93 \pm 0.02$ & $3.0 \pm 0.06$ & $0.51 \pm 0.01$ & $0.31 \pm 0.01$ & $0.28 \pm 0.01$ & $0.44 \pm 0.01$ & $0.36 \pm 0.01$ \\
\hline & & $60-70$ & $1.0 \pm 0.02$ & $25 \pm 0.84$ & $0.05 \pm 0.00$ & $0.04 \pm 0.00$ & $1.1 \pm 0.00$ & $3.5 \pm 0.09$ & $0.13 \pm 0.00$ \\
\hline
\end{tabular}

$9 \mathrm{Ox}=$ ammonium acid oxalate $(\mathrm{pH}=3), \mathrm{DCB}=$ dithionate-citrate-bicarbonate and $\mathrm{Na}$-py $=$ sodium pyrophosphate $(\mathrm{pH}=10)$. 

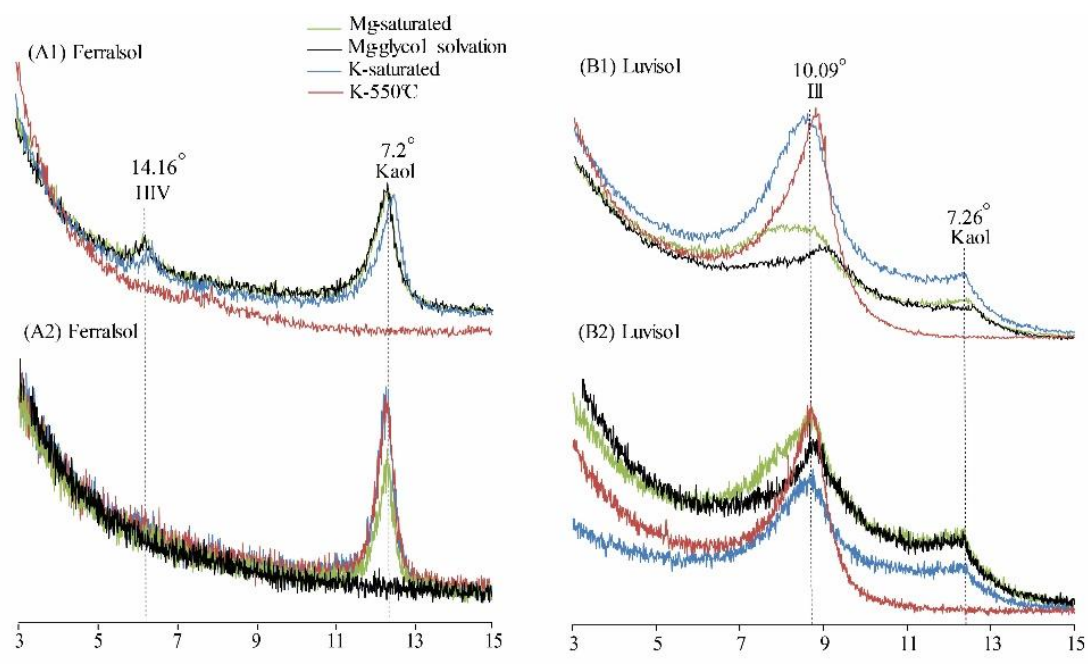

(C1) Vertisol

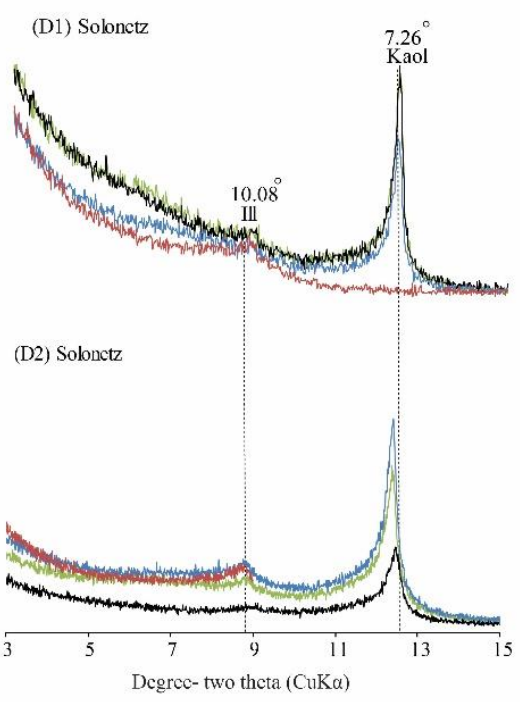

Fig. S1 X-ray diffraction analysis of the oriented clay fraction of four surface $(0-10 \mathrm{~cm})$ bulk soils from cropped (1) and native (2) land uses. HIV = hydroxyl interlayered vermiculite, $\mathrm{Ill}=$ illite, $\mathrm{Kaol}=$ kaolinite and $\mathrm{Sm}=$ smectite 

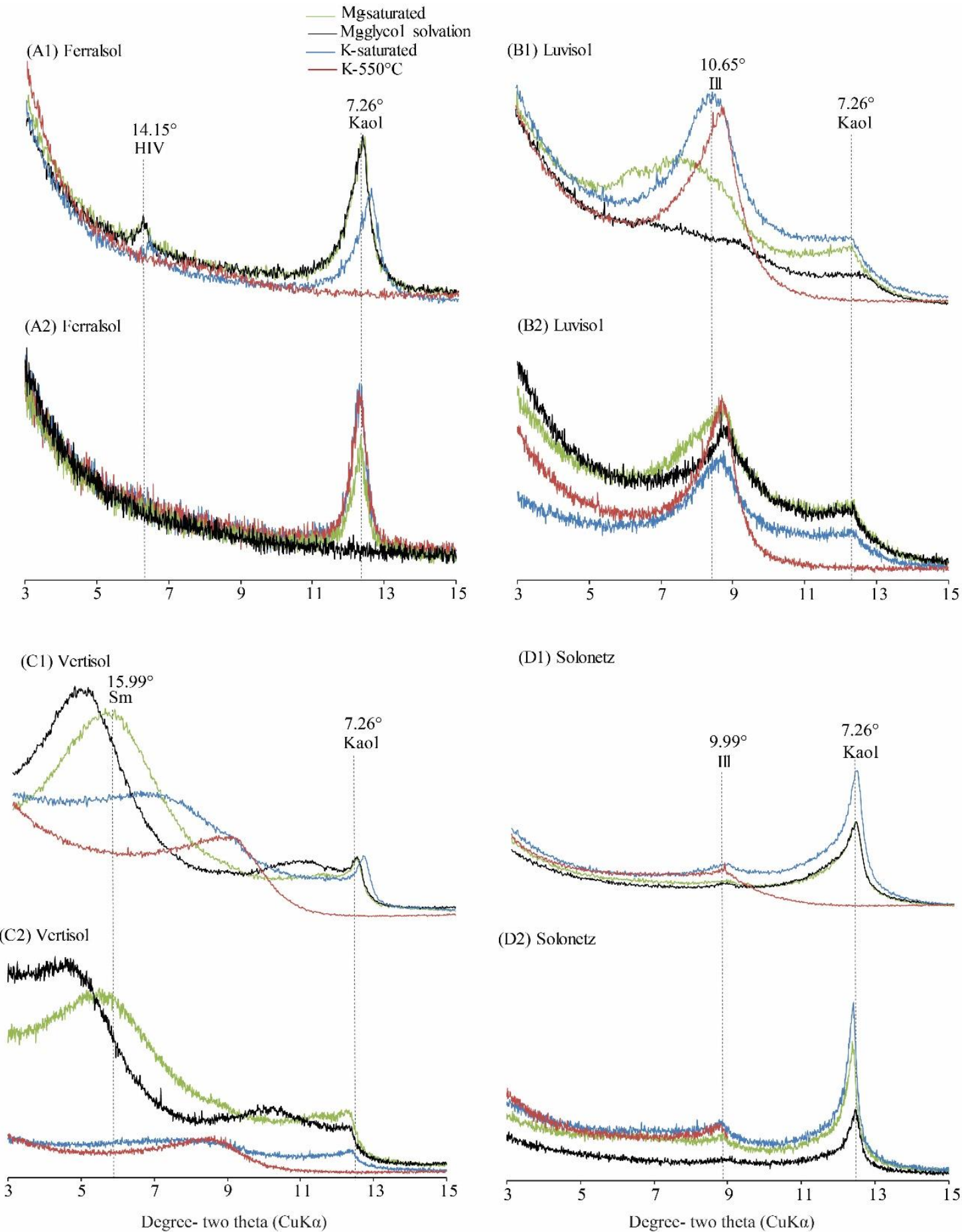

(D1) Solonetz

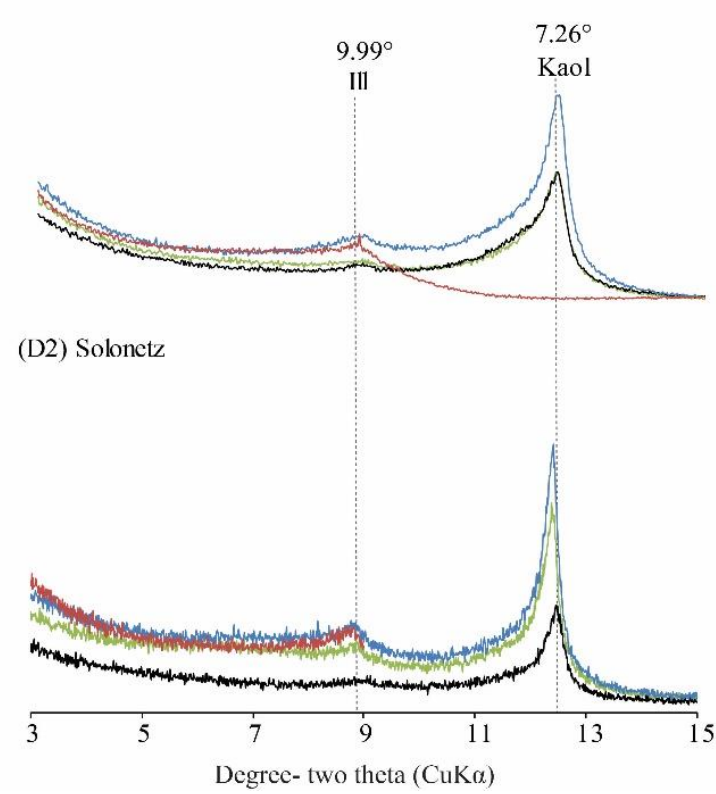

Fig. S2 X-ray diffraction analysis of the oriented clay fraction of four sub-surface (60-70 $\mathrm{cm}$ ) bulk soils from cropped (1) and native (2) land uses. HIV = hydroxyl interlayered vermiculite, $\mathrm{Ill}=$ illite, $\mathrm{Kaol}=$ kaolinite and $\mathrm{Sm}=$ smectite 
(A1) Ferralsol
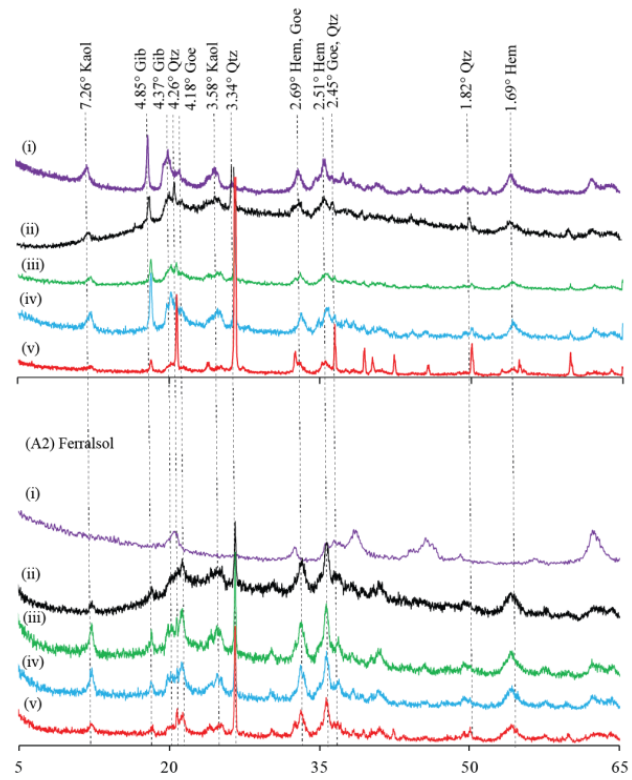

(C1) Vertisol
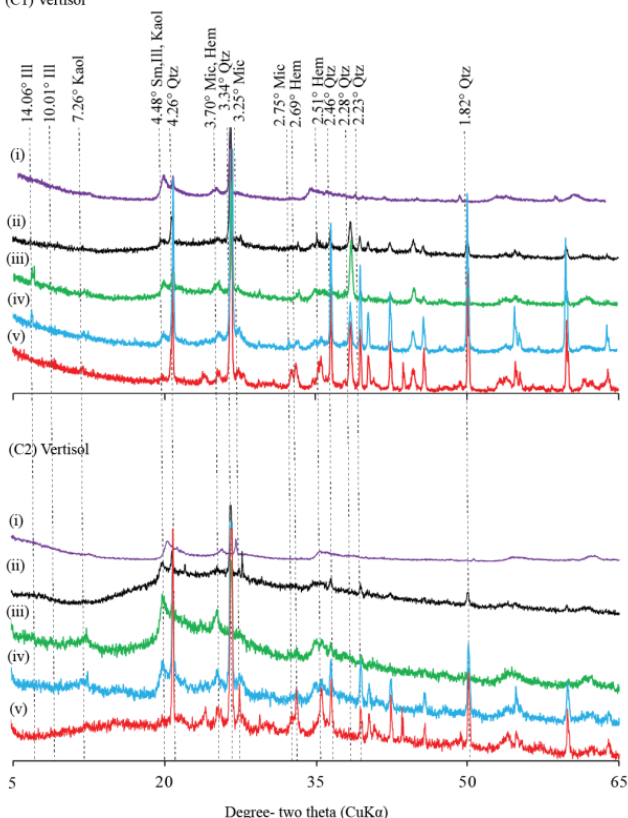

(B1) Luvisol

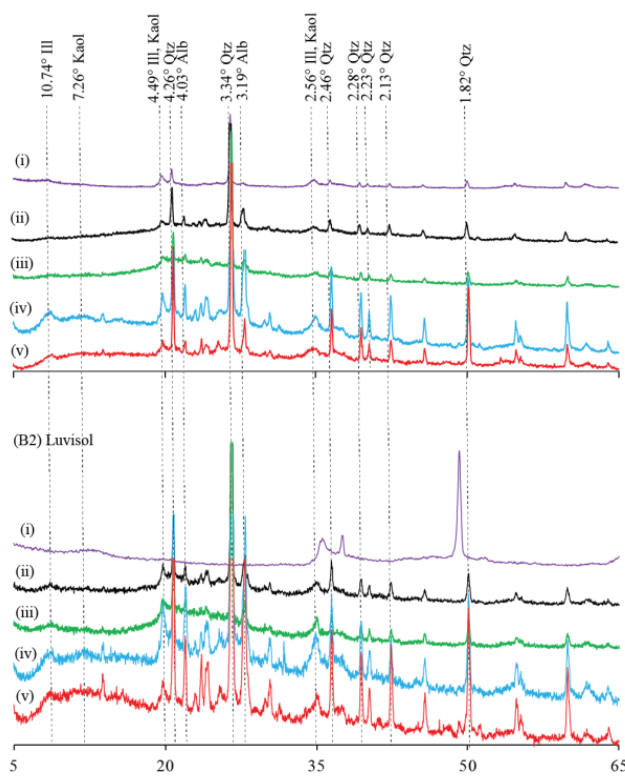

(D1) Solonetz
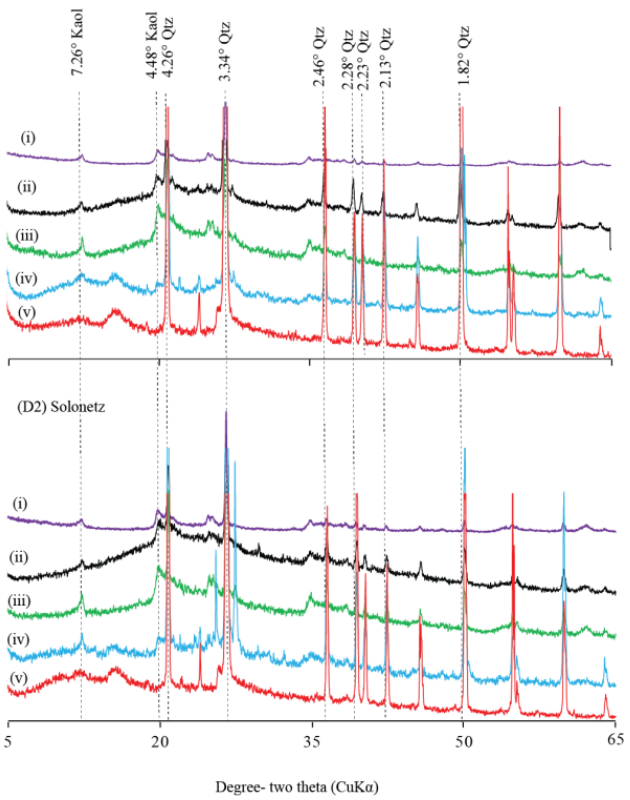

Fig. S3 X-ray diffraction analysis of the random powder of the bulk (i) and density fractions (ii $=\left\langle 1.8\right.$, iii $=1.8-2.2$, iv $=2.2-2.6$ and $\left.\mathrm{v}=>2.6 \mathrm{~g} \mathrm{~cm}^{-3}\right)$ of four surface $(0-10 \mathrm{~cm})$ soils from cropped (1) and native (2) land uses. Alb = albite, Gib = gibbsite, Goe = goethite, Hem $=$ hematite, Ill $=$ illite, Kaol $=$ kaolinite, Mic $=$ microcline, $\mathrm{Qtz}=$ quartz and Sm $=$ smectite 

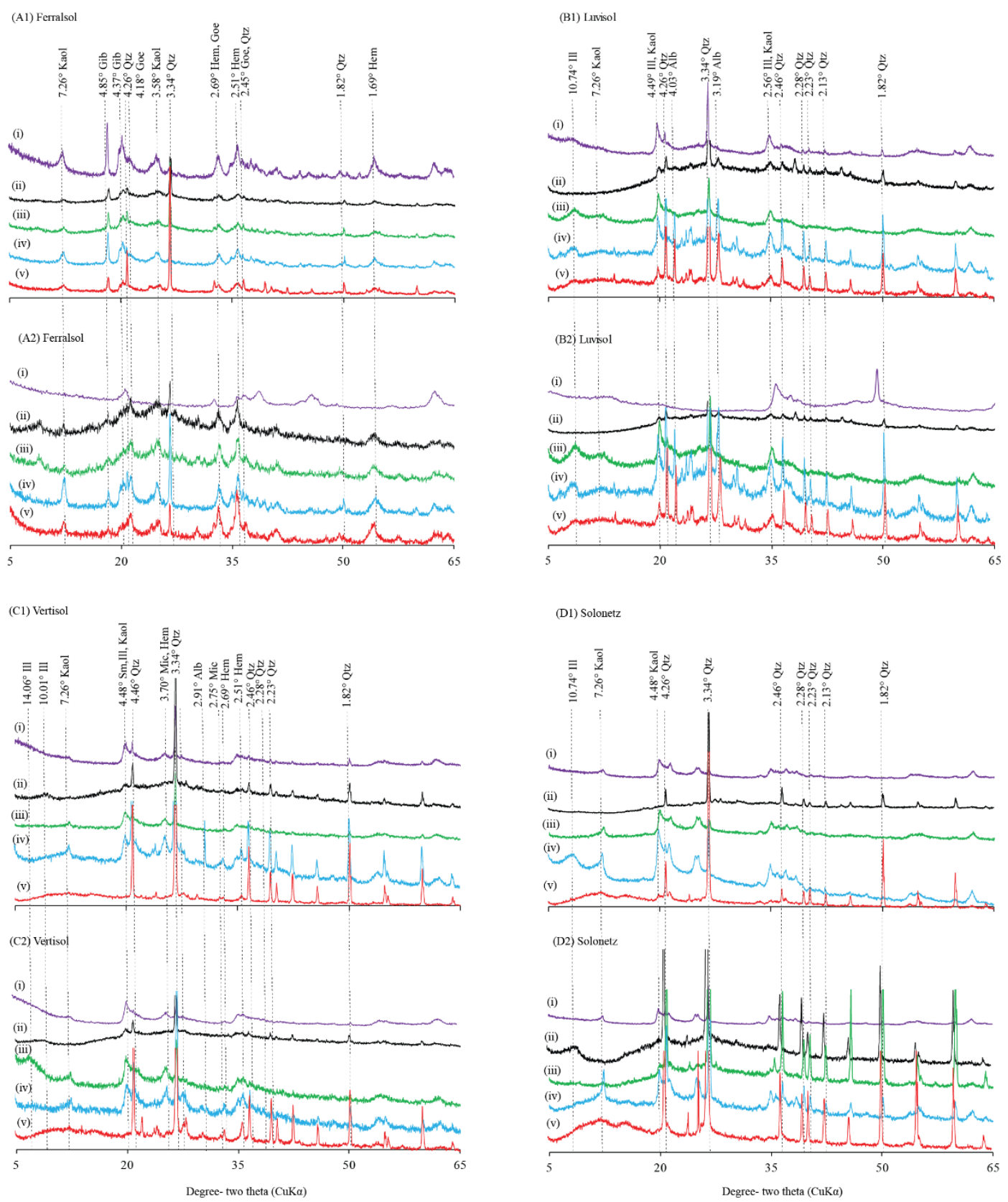

Fig. S4 X-ray diffraction analysis of the random powder of the bulk (i) and density fractions (ii $=\left\langle 1.8\right.$, iii $=1.8-2.2$, iv $=2.2-2.6$ and $\left.\mathrm{v}=>2.6 \mathrm{~g} \mathrm{~cm}^{-3}\right)$ of four sub-surface $(60-10 \mathrm{~cm})$ soils from cropped (1) and native (2) land uses. Alb = albite, Gib = gibbsite, Goe = goethite, Hem $=$ hematite, Ill $=$ illite, $\mathrm{Kaol}=$ kaolinite, Mic $=$ microcline, $\mathrm{Qtz}=$ quartz and $\mathrm{Sm}=$ smectite 


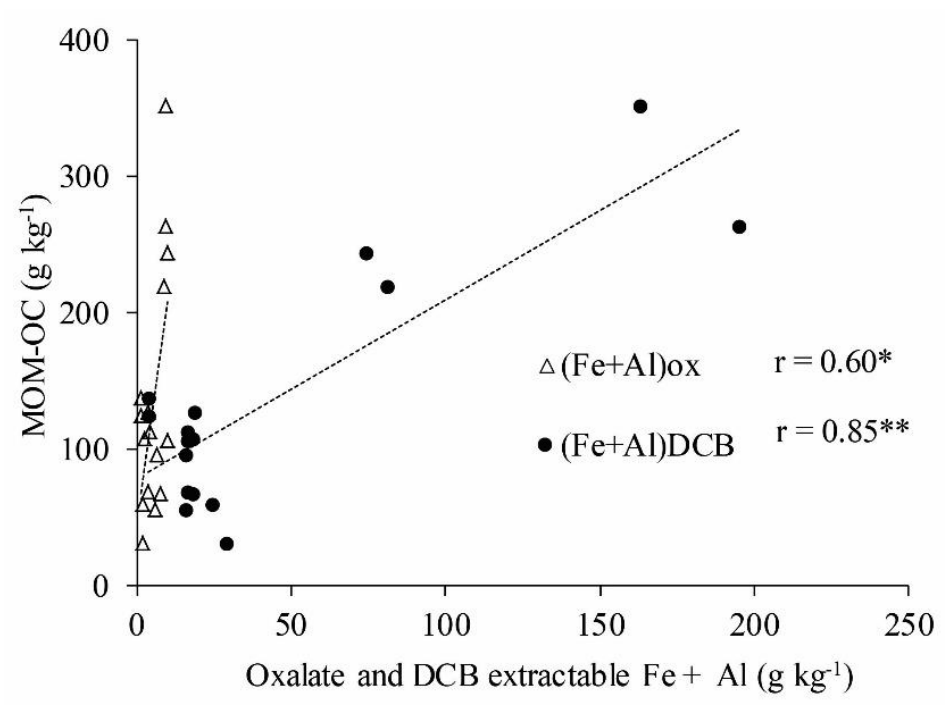

Fig. S5 Relation of the mineral associated organic matter (MOM, >1.8 $\left.\mathrm{g} \mathrm{cm}^{-3}\right)-\mathrm{OC}$ concentration of four soils with oxalate (ox) and $\mathrm{DCB}$ extractable $\mathrm{Fe}+\mathrm{Al}$ of the respective bulk soils, irrespective of soil depth and land use. * indicates significance at $p<0.05$ and ** indicates significance at $p<0.01$

\section{References}

Bureau of Meteorology (2014) Climate and past weather. Available online at: http://www.bom.gov.au/climate/. Accessed 17 May 2014

New South Wales Department of Primary Industries (2014) Climate at the wollongbar primary industries institute. http://www.dpi.nsw.gov.au/agriculture/resources/climate-and-weather. Assessed 17 May 2014 


\section{Raw Data}

Table 3. Proportion of total OC and total $\mathrm{N}$ in the density fractions of four surface $(0-10 \mathrm{~cm})$ and sub-surface $(60-70 \mathrm{~cm})$ soils under native and cropped land uses.

\begin{tabular}{|c|c|c|c|c|c|c|}
\hline \multirow{2}{*}{ Soil order } & \multirow{2}{*}{ Land use } & \multicolumn{4}{|c|}{ Proportion of total OC in the fractions } & \multirow{2}{*}{$\begin{array}{l}\text { Total fraction } \\
\text { recovery (\%) }\end{array}$} \\
\hline & & $\mathrm{POM}$ & $1.8 \mathrm{DF}$ & $2.2 \mathrm{DF}$ & $>2.6 \mathrm{DF}$ & \\
\hline \multicolumn{7}{|c|}{ Surface $(0-10 \mathrm{~cm})$} \\
\hline \multirow[t]{2}{*}{ Ferralsol } & Native & 7 & 15 & 30 & 43 & 95 \\
\hline & Cropped & 4 & 11 & 56 & 27 & 99 \\
\hline \multirow[t]{2}{*}{ Luvisol } & Native & 33 & 23 & 20 & 9 & 86 \\
\hline & Cropped & 40 & 26 & 32 & 1 & 99 \\
\hline \multirow[t]{2}{*}{ Vertisol } & Native & 10 & 45 & 35 & 3 & 93 \\
\hline & Cropped & 21 & 48 & 28 & 3 & 100 \\
\hline \multirow[t]{2}{*}{ Solonetz } & Native & 73 & 21 & 3 & 3 & 100 \\
\hline & Cropped & 44 & 29 & 12 & 2 & 87 \\
\hline \multicolumn{7}{|c|}{ Sub-surface $(60-70 \mathrm{~cm})$} \\
\hline \multirow[t]{2}{*}{ Ferralsol } & Native & 14 & 12 & 19 & 48 & 93 \\
\hline & Cropped & 7 & 5 & 46 & 38 & 96 \\
\hline \multirow[t]{2}{*}{ Luvisol } & Native & 17 & 12 & 47 & 7 & 83 \\
\hline & Cropped & 18 & 54 & 20 & 7 & 99 \\
\hline \multirow[t]{2}{*}{ Vertisol } & Native & 4 & 56 & 35 & 6 & 101 \\
\hline & Cropped & 14 & 39 & 38 & 9 & 99 \\
\hline \multirow[t]{4}{*}{ Solonetz } & Native & 16 & 5 & 41 & 23 & 84 \\
\hline & Cropped & 13 & 29 & 35 & 23 & 100 \\
\hline & & \multicolumn{4}{|c|}{ Proportion of total $\mathrm{N}$ in the fractions } & Total fraction \\
\hline & & $\mathrm{POM}$ & $1.8 \mathrm{DF}$ & $2.2 \mathrm{DF}$ & $>2.6 \mathrm{DF}$ & recovery $(\%)$ \\
\hline \multicolumn{7}{|c|}{ Surface $(0-10 \mathrm{~cm})$} \\
\hline \multirow[t]{2}{*}{ Ferralsol } & Native & 4 & 10 & 38 & 44 & 95 \\
\hline & Cropped & 3 & 9 & 67 & 15 & 93 \\
\hline \multirow[t]{2}{*}{ Luvisol } & Native & 21 & 20 & 27 & 15 & 82 \\
\hline & Cropped & 23 & 21 & 51 & 5 & 100 \\
\hline \multirow[t]{2}{*}{ Vertisol } & Native & 3 & 28 & 67 & 3 & 99 \\
\hline & Cropped & 6 & 41 & 48 & 5 & 101 \\
\hline \multirow[t]{2}{*}{ Solonetz } & Native & 49 & 23 & 6 & 4 & 83 \\
\hline & Cropped & 27 & 32 & 18 & 4 & 82 \\
\hline
\end{tabular}

Sub-surface $(60-70 \mathrm{~cm})$ 


\begin{tabular}{ccccccc}
\hline Ferralsol & Native & 2 & 3 & 22 & 53 & 80 \\
& Cropped & 7 & 5 & 46 & 42 & 100 \\
Luvisol & Native & 6 & 4 & 79 & 10 & 99 \\
& Cropped & 6 & 30 & 47 & 11 & 95 \\
& & & & & & \\
Vertisol & Native & 1 & 41 & 54 & 4 & 99 \\
& Cropped & 5 & 26 & 49 & 15 & 94 \\
& & & & & & 87 \\
Solonetz & Native & 4 & 3 & 60 & 20 & 99 \\
& Cropped & 3 & 14 & 61 & 21 & 97 \\
\hline
\end{tabular}

Density fractions: $\mathrm{POM}=<1.8 \mathrm{~g} \mathrm{~cm}^{-3}$ and MOM: $1.8 \mathrm{DF}=1.8-2.2 \mathrm{~g} \mathrm{~cm}^{-3}, 2.2 \mathrm{DF}=2.2-2.6 \mathrm{~g} \mathrm{~cm}^{-3}$ and $>2.6 \mathrm{DF}=$ $>2.6 \mathrm{~g} \mathrm{~cm}^{-3}$.

Table 4. Changes in $\mathrm{OC}$ and $\mathrm{N}$ concentrations in density fractions of four surface $(0-10 \mathrm{~cm})$ and sub-surface $(60-70 \mathrm{~cm})$ soils with land use conversion from native to cropped.

\begin{tabular}{cccccccccc}
\hline \multirow{2}{*}{ Soils } & \multirow{2}{*}{ Depth $(\mathrm{cm})$} & \multicolumn{3}{c}{ POM } & \multicolumn{2}{c}{$1.8 \mathrm{DF}$} & \multicolumn{2}{c}{$2.2 \mathrm{DF}$} & \multicolumn{2}{c}{$>2.6 \mathrm{DF}$} \\
\cline { 2 - 10 } & & $\mathrm{OC}$ & $\mathrm{N}$ & $\mathrm{OC}$ & $\mathrm{N}$ & $\mathrm{OC}$ & $\mathrm{N}$ & $\mathrm{OC}$ & $\mathrm{N}$ \\
\hline \multirow{2}{*}{ Ferrosol } & $0-10$ & -20 & -5 & -26 & -4 & -50 & -48 & -10 & -49 \\
& $60-70$ & -20 & -17 & -18 & -42 & 5 & -9 & -7 & -4 \\
Chromosol & $0-10$ & -5 & -5 & -8 & -8 & -30 & -24 & -17 & 28 \\
& $60-70$ & -7 & -5 & -37 & -1 & -34 & -4 & -19 & -7 \\
Vertosol & $0-10$ & -3 & -29 & -7 & -9 & -26 & -54 & -29 & -1 \\
& $60-70$ & -21 & 2 & -17 & -9 & -7 & -10 & -25 & 77 \\
Sodosol & $0-10$ & -8 & 1 & -8 & -2 & -18 & -31 & -22 & 26 \\
& $60-70$ & -27 & -16 & -48 & -42 & -45 & 11 & -5 & 25 \\
\hline Density fractions: POM $=<1.8 \mathrm{~g} \mathrm{~cm}^{-3}$ and MOM: $1.8 \mathrm{DF}=1.8-2.2 \mathrm{~g} \mathrm{~cm}^{-3}, 2.2 \mathrm{DF}=2.2-2.6 \mathrm{~g} \mathrm{~cm}^{-3}$ and $>2.6 \mathrm{DF}=$ \\
$>2.6 \mathrm{~g} \mathrm{~cm}^{-3}$.
\end{tabular}

Table 5. Change in isotopic values, i.e., $\Delta \delta^{13} \mathrm{C}$ and $\Delta \delta^{15} \mathrm{~N}$ in density fraction of four surface $(0-10 \mathrm{~cm})$ and sub-surface $(60-70 \mathrm{~cm})$ soils with land use change.

\begin{tabular}{cccccccccc}
\hline \multirow{2}{*}{ Soils } & \multirow{2}{*}{ Depth $(\mathrm{cm})$} & \multicolumn{2}{c}{ POM } & \multicolumn{2}{c}{$1.8 \mathrm{DF}$} & \multicolumn{2}{c}{$2.2 \mathrm{DF}$} & \multicolumn{2}{c}{$>2.6 \mathrm{DF}$} \\
\cline { 2 - 9 } & & ${ }^{13} \mathrm{C}$ & ${ }^{15} \mathrm{~N}$ & ${ }^{13} \mathrm{C}$ & ${ }^{15} \mathrm{~N}$ & ${ }^{13} \mathrm{C}$ & ${ }^{15} \mathrm{~N}$ & ${ }^{13} \mathrm{C}$ & ${ }^{15} \mathrm{~N}$ \\
\hline \multirow{2}{*}{ Ferralsol } & $0-10$ & 0.4 & -3.8 & 1.7 & -3.4 & 4.8 & -1.4 & 4.3 & -0.4 \\
& $60-70$ & -0.3 & -0.1 & 0.3 & 0.6 & 1.7 & 0.3 & 2.9 & 1.1 \\
Luvisol & $0-10$ & -1.5 & -1.9 & 0.0 & -2.0 & -0.5 & -1.0 & -0.6 & -1.0 \\
& $60-70$ & -1.6 & -1.7 & 0.0 & 3.3 & 0.1 & 0.8 & 0.8 & 1.1 \\
Vertisol & $0-10$ & 0.9 & 1.8 & 1.1 & 1.2 & 1.6 & 1.8 & 1.1 & 2.3 \\
& $60-70$ & 1.2 & 0.2 & 1.0 & -0.3 & 1.7 & 0.3 & 3.8 & 1.6 \\
Solonetz & $0-10$ & 4.6 & -0.3 & 1.3 & -0.6 & 1.5 & -0.2 & 1.5 & 7.4 \\
& $60-70$ & 0.1 & -0.7 & 0.7 & 2.3 & 1.7 & 4.3 & 2.3 & 4.5 \\
\hline fractions: POM $=<1.8 \mathrm{~g} \mathrm{~cm}^{-3}$ and MOM: $1.8 \mathrm{DF}=1.8-2.2 \mathrm{~g} \mathrm{~cm}^{-3}, 2.2 \mathrm{DF}=2.2-2.6 \mathrm{~g} \mathrm{~cm}^{-3}$ and $>2.6 \mathrm{DF}=$
\end{tabular}
$>2.6 \mathrm{~g} \mathrm{~cm}^{-3}$. 